\title{
Quantitative Assessment of Foveal Avascular Zone in Patients with Early and Intermediate Nonexudative Age-Related Macular Degeneration Using Optical Coherence Tomography-Angiography
}

\author{
Vladimir Stavrev, Nelly Sivkova, Desislava Koleva-Georgieva \\ Department of Ophthalmology, Faculty of Medicine, Medical University of Plovdiv, Plovdiv, Bulgaria \\ Email:vl_stavrev@abv.bg
}

How to cite this paper: Stavrev, V., Sivkova, N. and Koleva-Georgieva, D. (2018) Quantitative Assessment of Foveal Avascular Zone in Patients with Early and Intermediate Nonexudative Age-Related Macular Degeneration Using Optical Coherence Tomography-Angiography. Open Journal of Ophthalmology, 8, 133-139.

https://doi.org/10.4236/ojoph.2018.83017

Received: May 29, 2018

Accepted: July 22, 2018

Published: July 25, 2018

Copyright $\odot 2018$ by authors and Scientific Research Publishing Inc. This work is licensed under the Creative Commons Attribution International License (CC BY 4.0).

http://creativecommons.org/licenses/by/4.0/

\begin{abstract}
Optical coherence tomography-angiography (OCT-A) is a relatively new non-invasive imaging technique which provides three-dimensional visualization of the retinal and choroidal microvasculature. Recently, OCT-A is widely used in the diagnosis of age-related macular degeneration (AMD). Purpose: To compare 3 quantitative indexes of foveal avascular zone (FAZ) - area (A), perimeter $(\mathrm{Pm})$, and circularity $(\mathrm{C})$ in patients with early and intermediate nonexudative AMD and healthy subjects using OCT-A. Methods: Eighty-nine patients with nonexudative AMD ( $\mathrm{n}=89$ eyes) were enrolled in the study. They were compared with sex- and age-matched control group of 66 healthy subjects ( $\mathrm{n}=66$ eyes). The patients with nonexudative AMD were divided into 2 subgroups-early AMD ( $\mathrm{n}=42$ eyes) and intermediate AMD ( $\mathrm{n}=47$ eyes). All participants underwent OCT-A $(3 \times 3 \mathrm{~mm}$ scans, Cirrus HD-OCT, Angioplex, Carl Zeiss Meditec, Dublin, CA). Results: There was no statistically significant difference in all 3 assessed indexes between the early AMD subgroup and the control group $(\mathrm{A}-\mathrm{P}=0.139$; $\mathrm{Pm}-\mathrm{P}=0.230$; and $\mathrm{C}-\mathrm{P}=$ 0.191). There was also no statistically significant difference in all 3 assessed indexes between the intermediate AMD subgroup and the control group (A $\mathrm{P}=0.106, \mathrm{Pm}-\mathrm{P}=0.124$, and $\mathrm{C}-\mathrm{P}=0.102$, respectively). Conclusions: According to our results from the assessment of FAZ in patients with early and intermediate $\mathrm{AMD}$, we hypothesize that macular perfusion remains fairly unchanged in the nonexudative stages of the disease. OCT-A is a reliable technique for visualizing and quantifying $\mathrm{FAZ}$ in patients with nonexudative AMD.
\end{abstract}




\section{Keywords}

Age-Related Macular Degeneration, Optical Coherence

Tomography-Angiography, Foveal Avascular Zone

\section{Introduction}

Age-related macular degeneration (AMD) is the leading cause of visual impairment in individuals over the age of 55 years worldwide [1]. The clinical classification of AMD defines 3 stages according to the size of drusen, pigmentary abnormalities, and/or choroidal neovascularization (CNV) assessed within 2 disc diameters of the fovea-early, intermediate, and late AMD, including geographic atrophy (GA) and CNV [2].

The traditional multimodal imaging modalities in AMD include fluorescein angiography (FA), indocyanine green angiography (ICGA), and optical coherence tomography (OCT) [3]. OCT-angiography (OCT-A) is a relatively new non-invasive imaging technique. It provides three-dimensional visualization of the retinal and choroidal microvasculature, without the need of an intravenous dye [4]. A motion contrast is generated by differentiating between moving erythrocytes in the vessels and static surrounding tissue [5]. Each OCT-A-scan is simultaneously obtained along with the corresponding standard OCT B-scan. Thus, both functional and morphological images are acquired [6].

Recently, OCT-A has been used in many vascular and degenerative diseases (AMD, diabetic retinopathy, central/branch retinal vein occlusion, etc.) to visualize and examine microvasculature, as well as foveal avascular zone (FAZ), in different vascular plexuses separately [7] [8]. FAZ is a non-vascular area within the central part of the macula-fovea. Its dimensions vary among normal individuals [8] [9]. In addition, in various retinal diseases FAZ may be altered or deformed which may correlate with decreased best-corrected visual acuity (BCVA) [10] [11] [12].

The purpose of the recent study was to compare 3 quantitative indexes of the superficial FAZ-area (A), perimeter (Pm), and circularity (C) in a Caucasian cohort with early and intermediate nonexudative AMD, and healthy subjects using OCT-A.

\section{Materials and Methods}

This prospective study was approved by Ethics Committee of Medical University of Plovdiv, Bulgaria, and conducted in accordance with the requirements of Declaration of Helsinki. A total of 89 consecutive patients (48 female, 41 male) with nonexudative AMD ( $\mathrm{n}=89$ eyes) were enrolled in the study (Table 1$)$. The age (mean \pm standard deviation, SD) was $67.8 \pm 6.4$ years (range, $56-81$ ). According to the clinical classification of AMD, they were divided into 2 subgroups-early AMD with drusen $\geq 63-<125 \mu \mathrm{m}(\mathrm{n}=42$ eyes) and intermediate 
Table 1. Characteristics of the examined groups.

\begin{tabular}{ccccc}
\hline & $\begin{array}{c}\text { Early AMD } \\
(\mathrm{n}=42 \text { eyes })\end{array}$ & $\begin{array}{c}\text { Intermediate } \\
\text { AMD } \\
(\mathrm{n}=47 \text { eyes })\end{array}$ & $\begin{array}{c}\text { Control group } \\
(\mathrm{n}=66 \text { eyes })\end{array}$ & $\begin{array}{c}\text { Overall patients } \\
(\mathrm{n}=155 \text { eyes })\end{array}$ \\
\hline$\underline{\text { Age }}$ & & & & \\
\hline (mean \pm SD) Range & $66.5 \pm 6.1$ & $69 \pm 6.3$ & $64 \pm 4.8$ & $66 \pm 6$ \\
& $56-76$ & $57-81$ & $55-74$ & $55-81$ \\
\hline Gender & & & & 71 \\
Male & 20 & 21 & 30 & 84 \\
\hline Female & 22 & 26 & 36 & \\
\hline
\end{tabular}

$\mathrm{AMD}=$ age-related macular degeneration, $\mathrm{SD}=$ standard deviation.

AMD with drusen $\geq 125 \mu \mathrm{m}$ and/or pigmentary abnormalities $(\mathrm{n}=47$ eyes). Criteria for inclusion in AMD patients group were age $\geq 55$ years old, drusen and/or retinal pigment epithelium (RPE) abnormalities (hyper-/hypopigmentation) secondary to AMD, a spherical equivalent of $\geq-3 \mathrm{D}$ to $\leq+3 \mathrm{D}$. Exclusion criteria were presence of CNV, GA, and other ocular degenerative, vascular or traumatic diseases. They were compared with sex- and age-matched control group of 66 healthy subjects ( $\mathrm{n}=66$ eyes, 36 female and 30 male).The age was 64 \pm 4.8 years, range $55-74$. The inclusion criteria for the healthy volunteers were age $\geq 55$ years old, BCVA of $\geq 1.0$, and no history or evidence of ocular or systemic disease.

All participants underwent a complete ophthalmologic examination, including BCVA (Snellen charts), slit-lamp biomicroscopy with fundus examination, tonometry, and OCT-A (Cirrus HD-OCT, Angioplex, Carl Zeiss Meditec, Dublin, CA). OCT-A has automatic algorithms that provide estimates of the "nonflow" area in the center of the macula, defining the FAZ (Figure 1). Quantitative analyses of the FAZ were performed using the $3 \times 3-\mathrm{mm}$ en face OCT-A images. In this study we used only the preset settings for the superficial FAZ because of software limitations. We measured 3 indexes of the superficial FAZ-A in $\mathrm{mm}^{2}$, Pm in $\mathrm{mm}$, and C. OCT-A scans with low quality of images were excluded. Written informed consent was obtained from all participants.

All statistical analyses were performed using statistical software (IBM SPSS software Version 19; SPSS, Inc., Chicago, IL, USA). The correlation between healthy subjects and each AMD subgroup was generated by independent-samples T-test. $\mathrm{P}$ values less than $\mathrm{P}<0.05$ were considered statistically significant.

\section{Results}

Our study analyzed 89 eyes of AMD patients and 66 eyes of healthy volunteers $(n=155$ eyes, $66 \pm 6$ years, range: $55-81)$. In the early AMD subgroup the following FAZ values (mean $\pm \mathrm{SD}$ ) were established: $\mathrm{A}-0.27 \pm 0.09 \mathrm{~mm}^{2}, \mathrm{Pm}-2.28$ $\pm 0.55 \mathrm{~mm}$, and $\mathrm{C}-0.66 \pm 0.08$. In the intermediate AMD subgroup the registered values were: $\mathrm{A}-0.28 \pm 0.08 \mathrm{~mm}^{2}, \mathrm{Pm}-2.28 \pm 0.34 \mathrm{~mm}$, and $\mathrm{C}-0.65 \pm$ 0.04 , and in the control group: $\mathrm{A}-0.24 \pm 0.08 \mathrm{~mm}^{2}, \mathrm{Pm}-2.16 \pm 0.44 \mathrm{~mm}$, and $\mathrm{C}-0.68 \pm 0.05$, respectively. The values are summarized in Table 2 . There was no statistically significant difference in all 3 assessed indexes between the early 

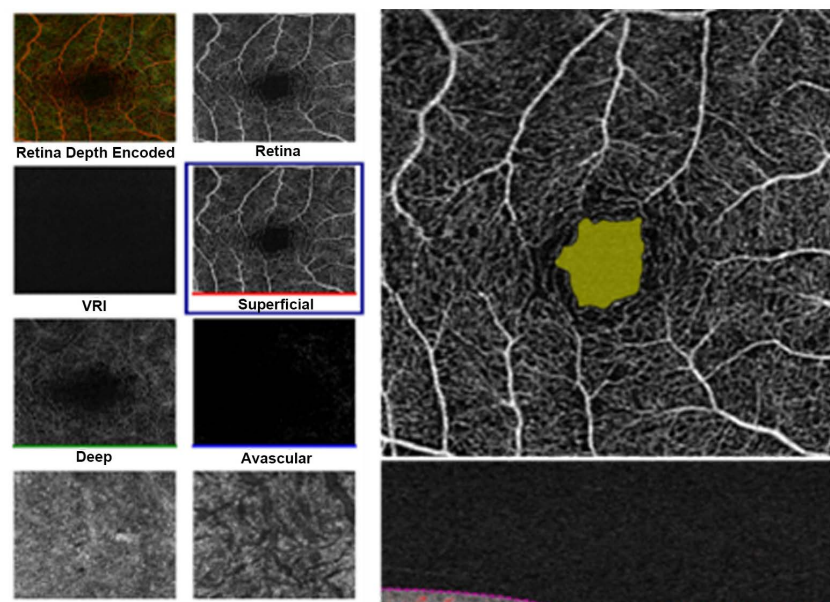

Choriocapillaris

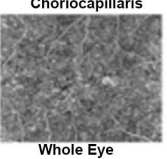

Choroid
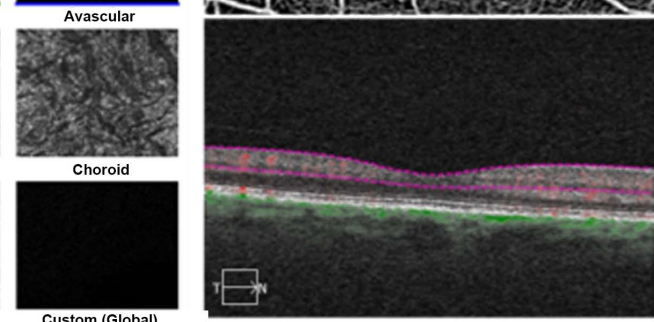

(a)

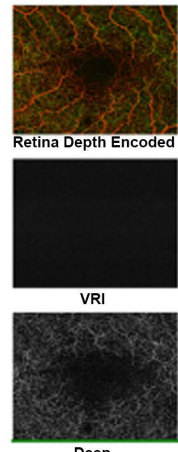

Deep

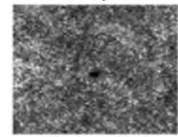

Choriocapillaris

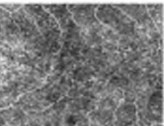

Whole Eye
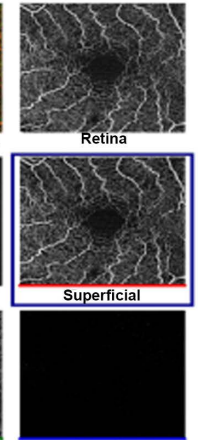

Avascular

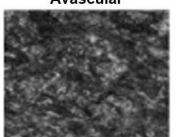

Choroid

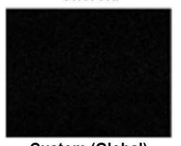

Custom (Global)
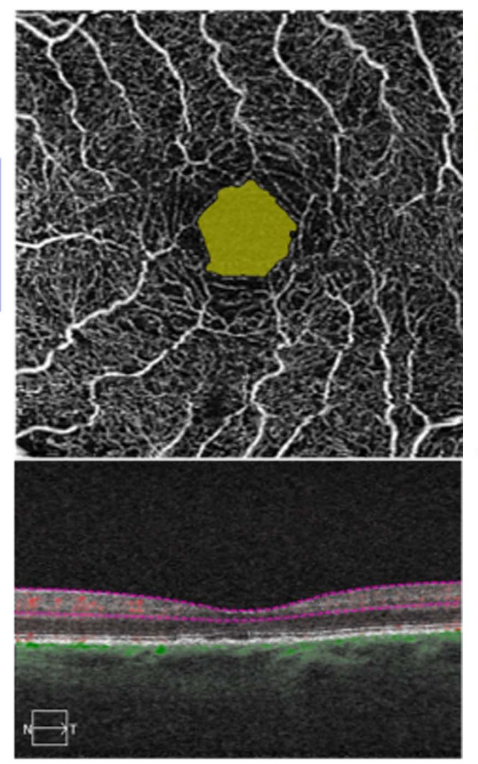

(b)

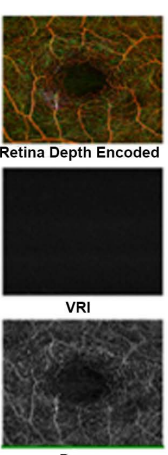

Deep

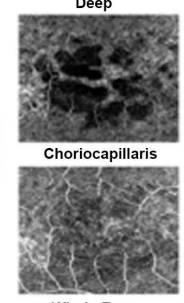

Whole Eye

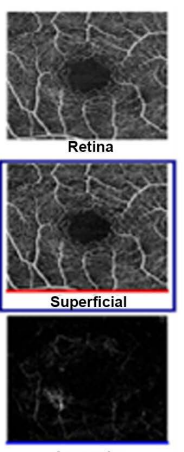

Avascular
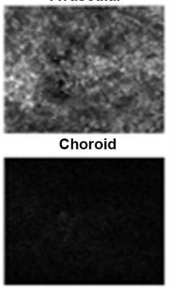

Custom (Global)
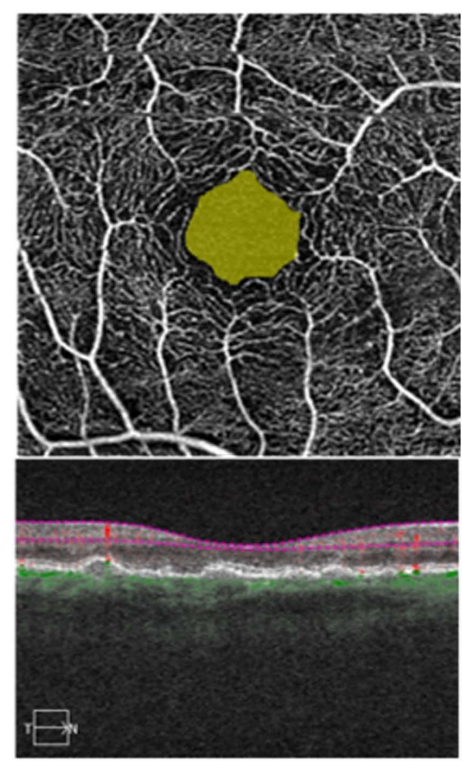

(c)

Figure 1. Superficial foveal avascular zone in healthy subject (a), in early AMD (b), and in intermediate AMD (c).

AMD subgroup and the control group: $\mathrm{A}-\mathrm{P}=0.139, \mathrm{Pm}-\mathrm{P}=0.230$, and $\mathrm{C}-\mathrm{P}=$ 0.191 . No statistically significant correlation was found in all 3 assessed indexes between the intermediate AMD subgroup and the control group as well: $\mathrm{A}-\mathrm{P}=$ $0.106, \mathrm{Pm}-\mathrm{P}=0.124$, and $\mathrm{C}-\mathrm{P}=0.102$, respectively.

\section{Discussion}

OCT-A enables precise evaluation and quantification of the retinal microvasculature at different levels, without the need of a dye. OCT-A has automatic algorithms defining the FAZ. A relation of FAZ with the age and gender in normal 
Table 2. Quantitative indexes of superficial foveal avascular zone (FAZ) in early and intermediate age-related macular degeneration (AMD), and in control group.

\begin{tabular}{cccc}
\hline $\begin{array}{c}\text { Superficial } \\
\text { FAZ indexes }\end{array}$ & $\begin{array}{c}\text { Early AMD } \\
(\mathrm{n}=\mathbf{4 2} \text { eyes })\end{array}$ & $\begin{array}{c}\text { Intermediate } \\
\text { AMD } \\
(\mathrm{n}=47 \text { eyes })\end{array}$ & $\begin{array}{c}\text { Control group } \\
(\mathrm{n}=66 \text { eyes })\end{array}$ \\
\hline Area $\left(\mathrm{mm}^{2}\right)$ & $0.27 \pm 0.09$ & $0.28 \pm 0.08$ & $0.24 \pm 0.08$ \\
Perimeter $(\mathrm{mm})$ & $2.28 \pm 0.55$ & $2.28 \pm 0.34$ & $2.16 \pm 0.44$ \\
Circularity & $0.66 \pm 0.08$ & $0.65 \pm 0.04$ & $0.68 \pm 0.05$ \\
\hline
\end{tabular}

eyes has been previously described by various studies. Samara et al. [8] and Tan et al. [13] reported no correlation between FAZ area and age in normal eyes whereas Iafe et al. [14] found a significant difference in the examined variables. In our study we did not find any statistically significant correlation between age and FAZ area in the group of healthy volunteers $(\mathrm{P}>0.354)$. FAZ area and gender remain a controversial topic which has to be confirmed or rejected by additional studies [8] [13].

In the analysis of superficial FAZ and examined parameters (A, Pm, and $\mathrm{C})$ on $3 \times 3-\mathrm{mm}$ scans, our results did not show any statistically significant difference between patients with different stages of nonexudative AMD and control group of healthy volunteers. According to that, we hypothesize that FAZ area and shape, as well as macular perfusion remain fairly unchanged in the nonvascular form of the disease. As far as we know this is the first study which compares FAZ variables such $\mathrm{A}, \mathrm{Pm}$, and $\mathrm{C}$ in patients with nonexudative AMD using OCT-A.

There are some limitations in this study. We did not evaluate deep FAZ because of the software version limitations. In all conducted studies deep FAZ was measured with a wider range compared to superficial FAZ [9] [14] [15]. Furthermore, central foveal thickness and axial length were not included in the overall assessment. Some authors describe negative correlation of superficial FAZ area with central retinal thickness [8] [13].

\section{Conclusion}

In conclusion, OCT-A is an appropriate non-invasive and reliable method for evaluating superficial FAZ that provides automatic quantitative measurement of this "nonflow" area in the center of the macula. According to our results, we hypothesize that macular perfusion remains fairly unchanged in the nonexudative stages of the disease. Additional studies are needed to confirm its application and importance in nonexudative stages of AMD.

\section{Conflict of Interest}

The authors declare no conflict of interest.

\section{Funding}

This publication is funded by Medical University of Plovdiv, Bulgaria (Project № 001/2017). 


\section{References}

[1] Wong, W.L., Su, X., Li, X., et al. (2014) Global Prevalence of Age-Related Macular Degeneration and Disease Burden Projection for 2020 and 2040: A Systematic Review and Meta-Analysis. The Lancet Global Health, 2, e106-e116. https://doi.org/10.1016/S2214-109X(13)70145-1

[2] Ferris III, F.L., Wilkinson, C.P., Bird, A., et al. (2013) Clinical Classification of Age-Related Macular Degeneration. Ophthalmology, 120, 844-851.

https://doi.org/10.1016/j.ophtha.2012.10.036

[3] Sulzbacher, F., Kiss, C., Munk, M., et al. (2011) Diagnostic Evaluation of Type 2 (classic) Choroidal Neovascularization: Optical Coherence Tomography, Indocyanine Green Angiography, an Fluorescein Angiography. American Journal of Ophthalmology, 152, 799-806 e1.

[4] Lumbroso, B., Huang, D., Jia, Y., et al. (2015) Clinical Guide to Angio-Oct Non Invasive, Dyeless OCT Angiography. 1st Edition, Jaypee Brothers Medical Publisher (P) Ltd., New Delhi.

[5] Jia, Y., Tan, O., Tokayer, J., et al. (2012) Split-Spectrum Amplitude-Decorrelation Angiography with Optical Coherence Tomography. Optics Express, 20, 4710-4725. https://doi.org/10.1364/OE.20.004710

[6] Jia, Y., Bailey, S.T., Wilson, D.J., et al. (2014) Quantitative Optical Coheren Cetomography Angiography of Choroidal Neovascularizationin Age-Related Macular Degeneration. Ophthalmology, 121, 1435-1444.

https://doi.org/10.1016/j.ophtha.2014.01.034

[7] Spaide, R.F., Klancnik, J.M. and Cooney, M.J. (2015) Retinal Vascular Layers Imaged by Fluorescein Angiography and Optical Coherence Tomography Angiography. JAMA Ophthalmology, 133, 45-50.

[8] Samara, W.A., Say, E.A.T., Khoo, C.T.L., Higgins, T.P., Magrath, G., Ferenczy, S., et al. (2015) Correlation of Foveal Avascular Zone Size with Foveal Morphology in normal Eyes Using Optical Coherence Tomography Angiography. Retina, 35, 2188-2195.

[9] Carpineto, P., Mastropasqua, R., Marchini, G., Toto, L., Di Nicola, M. and DiAntonio, L. (2016) Reproducibility and Repeatability of Foveal Avascular Zone Measurements in Healthy Subjects by Optical Coherence Tomography Angiography. British Journal of Ophthalmology, 100, 671-676.

[10] Coscas, F., et al. Optical Coherence Tomography Angiography in Retinal Vein Occlusion: Evaluation of Superficial and Deep Capillary Plexa. American Journal of Ophthalmology, 161, e161-e162.

[11] Balaratnasingam, C., et al. (2016) Visual Acuity Is Correlated with the Area of the Foveal Avascular Zone in Diabetic Retinopathy and Retinal Vein Occlusion. Ophthalmology, 123, 2352-2367. https://doi.org/10.1016/j.ophtha.2016.07.008

[12] Al-Sheikh, M., Akil, H., Pfau, M. and Sadda, S.R. (2016) Swept-Source OCT Angiography Imaging of the Foveal Avascular Zone and Macular Capillary Network Density in Diabetic Retinopathy. Investigative Ophthalmology \& Visual Science, 57, 3907-3913. https://doi.org/10.1167/iovs.16-19570

[13] Tan, C.S., Lim, L.W., Chow, V.S., et al. (2016) Optical Coherence Tomography Angiography Evaluation of the Parafoveal Vasculature and Its Relationship with Ocular Factors. Investigative Ophthalmology \& Visual Science, 57, OCT224-OCT234. https://doi.org/10.1167/iovs.15-18869

[14] Iafe, N.A., Phasukkijwatana, N., Chen, X. and Sarraf, D. (2016) Retinal Capillary 
Density and Foveal Avascular Zone Area Are Age-Dependent: Quantitative Analysis Using Optical Coherence Tomography Angiography. Investigative Ophthalmology \& Visual Science, 57, 5780-5787. https://doi.org/10.1167/iovs.16-20045

[15] Shahlaee, A., Pefkianaki, M., Hsu, J. and Ho, A.C. (2016) Measurement of Foveal Avascularzone Dimensions and Its Reliability in Healthy Eyes Using Optical Coherence Tomography Angiography. American Journal of Ophthalmology, 161, 50e55 e1. 